\title{
Sovente, di sovente, soventemente (e spesso): che differenza c'è?
}

\author{
Massimo Cerruti
}

PUBBLICATO: 22 NOVEMBRE 2019

\section{Quesito:}

Alcuni lettori ci chiedono se sia corretto l'uso di soventemente e di sovente, e che differenza ci sia tra queste due forme e sovente.

Sovente, di sovente, soventemente (e spesso): che differenza c'è?

$\mathrm{T}$

utte e tre le forme, sovente, di sovente e soventemente, sono attestate in dizionari dellitaliano (si vedano, fra gli altri, GDLI, GRADIT e Vocabolario Treccani) e, in funzione avverbiale, hanno lo stesso significato di spesso ed espressioni equivalenti. Il GDLI, ad esempio, glossa sovente e di sovente come "spesso, frequentemente, di frequente, ripetutamente, assiduamente, più volte" (s.v. sovénte) e soventemente come "spesso, di frequente, in molti casi, più volte" (s.v. soventeménte).

Sovente può poi comparire anche come aggettivo, nel senso di 'frequente' (es. le piogge sono soventi); proprio a partire dall'aggettivo sovente sono formati l'avverbio soventemente e la locuzione avverbiale di sovente.

Nell'uso avverbiale non vi sono dunque differenze di significato referenziale fra sovente, di sovente e soventemente, cosi come tra queste forme e un sinonimo quale spesso. Si hanno invece delle diversità in termini di frequenza e situazioni d'uso.

Il GRADIT pone spesso nel novero delle circa 2.000 parole di altissima frequenza che costituiscono il cosiddetto "vocabolario fondamentale" dell'italiano, mentre include sovente, come avverbio, fra i vocaboli di uso comune, che "sono usati e compresi indipendentemente dalla professione [...] o dalla collocazione regionale e che sono generalmente noti a chiunque abbia un livello mediosuperiore di istruzione" (Tullio De Mauro, Introduzione, in GRADIT, vol. I, pag. XX), e soventemente tra i vocaboli di basso uso, "rari, tuttavia circolanti ancora con qualche frequenza in testi e discorsi del Novecento" (ibidem); considera invece obsoleto l'impiego di sovente come aggettivo e non prende in esame la locuzione di sovente.

Per una prima idea della diversa frequenza d'uso di queste forme, anche in diacronia, possiamo dare uno sguardo con Ngram Viewer all'archivio di opere in italiano di Google Libri. Il grafico sottostante, che rappresenta la distribuzione percentuale delle forme considerate nei libri editi fra il i86r e il 2000 presenti in archivio, illustra il divario d'uso fra sovente, di sovente e soventemente, da un lato, e spesso, dall'alto, e mostra oltretutto come nell'arco di tempo osservato esso si sia fatto progressivamente più spiccato. 


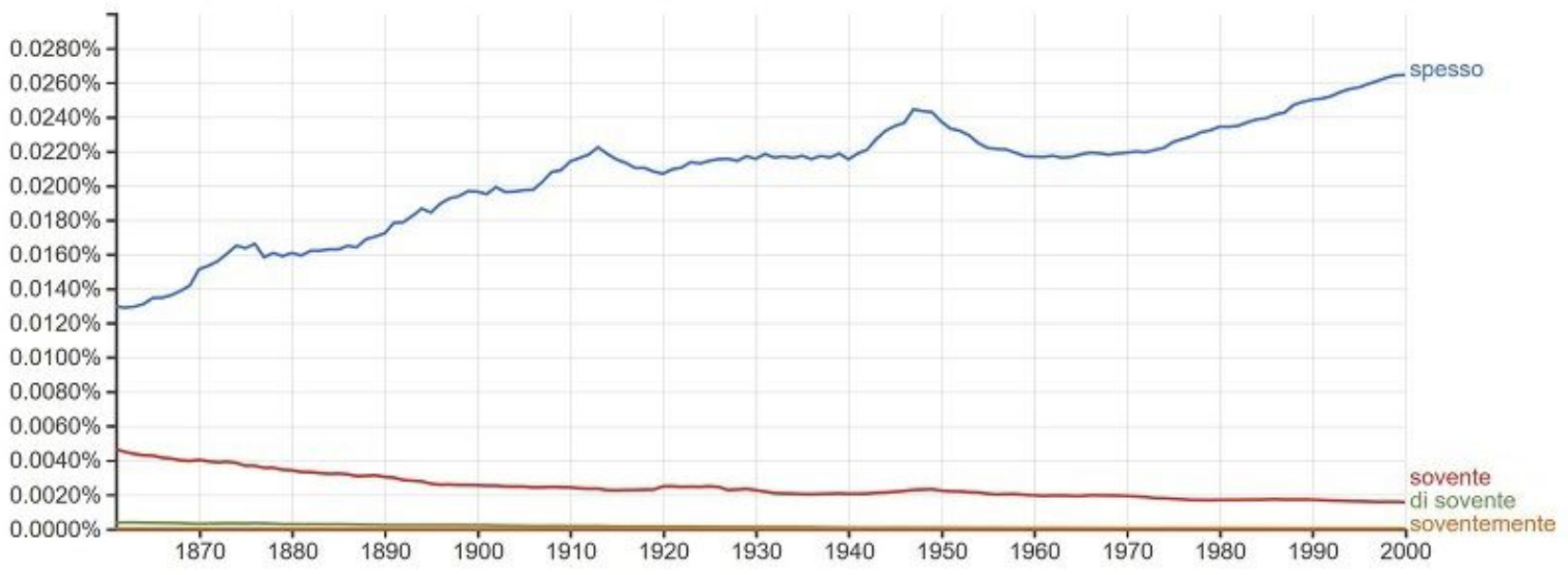

Le differenze riscontrabili in termini di frequenza d'uso sono poi da porre in relazione ai diversi contesti situazionali e tipi di testo nei quali queste forme compaiono più regolarmente. È facile notare come spesso sia un termine dell'uso medio, non marcato, proprio di parlanti con diversa collocazione sociale e "adatto" a più situazioni comunicative, mentre la presenza di sovente ("sentito come letterario e ricercato", Vocabolario Treccani, s.v. sovènte), di sovente e soventemente ("antico e letterario", GDLI, s.v. soventeménte) è piuttosto legata allo scritto e al parlato formale, specie di parlanti istruiti. Se ne dànno qui alcuni esempi tratti dalla prosa colta, di genere scientifico, saggistico e narrativo: "come di sovente accade nelle pagine dello studioso tedesco" (Laura D'Amati, A proposito del curator bonorum captivi, "Bollettino dell'Istituto di diritto romano Vittorio Scialoja", 42/43 (2000/200I), pp. 223-246: 228); "restavano a presidiare il territorio spagnolo, sovente lasciati senza paga" (Davide Maffi, La cittadella in armi: esercito, societá e finanza nella Lombardia di Carlo II, Milano, Franco Angeli, 20ro, p. 64); "soventemente accade nella vita che in qualche spiacevole occasione ci si trovi di fronte ad un delicato dilemma” (Romano Battaglia, Come si fa, Milano, Rizzoli, 2006, p. I55).

Ciò non di meno, forme come sovente possono avere un impiego più generalizzato in certi ambiti regionali, anche per effetto dell'influenza delle varietà di sostrato. Nei dialetti di area settentrionale, e specialmente del Nord-Ovest, forme quali spess e de spess, equivalenti all'italiano spesso, coesistono con forme come sovens e sovent, dello stesso tipo dellitaliano sovente (v. ad es. Gran dizionario piemonteseitaliano di Vittorio di Sant'Albino, Torino I859, rist. Savigliano I993, s.vv. spess e soenss; Dizionario monferrino di Sergio Nebbi, Savigliano 200I, s.vv. spës e suens; Vocabolario milanese-italiano di Francesco Cherubini, Milano I839-I856, rist. Cosenza 1959, s.vv. spèss e sovénz; Lessico dialettale della Svizzera italiana, Bellinzona 2004, s.vv. spéss e sovént), ma sono le seconde a risultare le più diffuse. Se guardiamo la carta 704 andava spesso dell'Atlante linguistico ed etnografico dell'Italia e della Svizzera meridionale (Sprach- und Sachatlas Italiens und der Südschweiz), possiamo infatti notare come in area settentrionale, e più in particolare nord-occidentale, le forme dialettali equivalenti a spesso siano nella maggior parte dei casi riconducibili a sovens e sovent; è così, ad esempio, in Piemonte e Valle d'Aosta (per varietà sia galloitaliche che galloromanze), nell'Imperiese, nel Pavese e in alcune regioni del Canton Ticino e dei Grigioni.

È dunque prevedibile che questa preferenza per il tipo sovente, riscontrabile nelle abitudini d'impiego del dialetto, possa essere trasferita all'uso dell'italiano (per via diretta, nei parlanti nativi di dialetto, o per trasmissione generazionale). Non a caso, nell'area in questione la presenza di sovente non è limitata allo scritto colto e al parlato formale. Si vedano ad esempio le occorrenze seguenti, l'una di ambito ligure e l'altra di ambito piemontese, rinvenibili rispettivamente nella scrittura di semicolti e nell'italiano (regionale popolare) parlato: "gioivano al vedere sovente qualche italiano a quelle torture" (Graziano Mamone, Le scritture dei prigionieri italiani nella Grande Guerra, in "Vegueta. Anuario de la 
Facultad de Geografía e Historia" i9 (2019), pp. 307-337: 331), "da inverno vado sovente in montagna" (Massimo Cerruti, Strutture dellitaliano regionale. Morfosintassi di una varieta diatopica in prospettiva sociolinguistica, Frankfurt am Main, Lang, 2009, p. 276). Sovente compare inoltre nella scrittura scolastica: nel grafico sottostante (riprodotto, con modifiche, da Luisa Revelli, Diacronia dellitaliano scolastico, Aracne, Roma 2013, p. 245) si può ad esempio vedere il risultato, in valori assoluti, dello spoglio di un archivio di 550 quaderni di scuola elementare redatti in Valle d'Aosta fra l'Unità d'Italia e la fine del XX secolo; il diagramma rivela tuttavia come l'iniziale preminenza di sovente abbia presto ceduto il passo al predominio di spesso, che, sulle prime lieve, si è fatto via via più sensibile a partire dalla metà del Novecento.

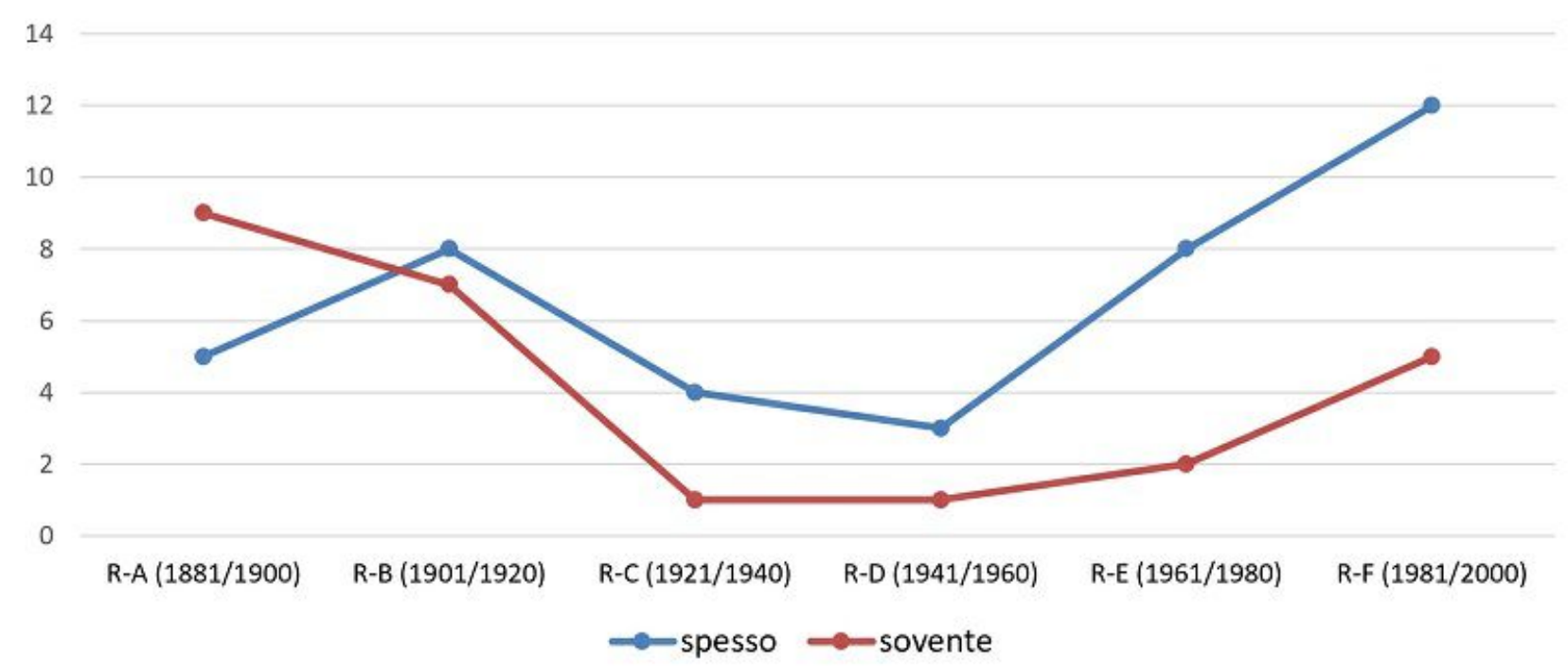

In conclusione, sovente, di sovente, soventemente (e spesso) non differiscono tra di loro sul piano del significato referenziale, essendo a tutti gli effetti sinonimi, ma mostrano diversità d'uso significative in relazione ai caratteri del contesto situazionale e del tipo di testo e alla provenienza geografica e collocazione sociale dei parlanti.

\section{Cita come:}

Massimo Cerruti, Sovente, di sovente, soventemente (e spesso): che differenza c'è?, "Italiano digitale", 2019, XI, 2019/4 (ottobre-dicembre)

DOI: $10.35948 / 2532-9006 / 2020.3252$

\section{Copyright 2019 Accademia della Crusca}

Pubblicato con licenza creative commons CC BY-NC-ND 